[3] K. Kirkopulo. "Razrabotka interfejsa operatora komp'juternoj sistemy avtomatizacii ustanovki ionno-plazmennogo napylenija": Avtomatizacija tehnologicheskih i biznes-processov, vol. 10, no. 4, pp.54-61, 2018.

[4] J. Rapoport, Ju. Pleshivceva. Problemy i metody teorii i tehniki optimal'nogo upravlenija sistemami s raspredelennymi parametrami. URL: http://www.ssc.smr.ru/media/ipuss_conf/15/2_01.pdf (last visited: 10.5.2019).

[5] A. Butkovskij. Metody upravlenija sistemami s raspredelennymi parametrami. Moscow: Nauka, 1975.

[6] O. Stopakevych, O. Ulitska. "Design of precise control systems of industrial plants", Eastern-European Journal of Enterprise Technologies, vol. 2, no. 2, pp.56-62, 2017.

[7] V. Tonkonogij, G. Oborskij, "Rabotosposobnost' i nadezhnost' instrumentov s iznosostojkimi pokrytijami”, Trudy Odesskogo politehnicheskogo universiteta, vol.7, no.1, pp.130-133, 1997.

[8] A. Butkovskij. Harakteristiki sistem s raspredelennymi parametrami. Moscow: Nauka, 1979.

[9] I. Butusov, A. Kumakov, V. Hudikovskiy, Ju. Perin. 'Raschet raspredelenija temperatury vdol' sterzhnja pri nestacionarnom teploobmene metodom lokal'nyh teplovyh potokov", Nauchnyj vestnik Voronezhskogo gosudarstvennogo arhitekturno-stroitel'nogo universiteta. Stroitel'stvo i arhitektura, vol. 25, no. 1, pp. 50-57, 2012.

[10] A. Kudajkulov, B. Kenzhegulov, U. Utebaev, V. Tokkuliev. "Chislennoe modelirovanie termo-naprjazhennogo sostojanija sterzhnja v vide usechennogo konusa”. Problemy informatiki, no. 4, pp. 14-21, 2015.

[11] Ju. Vidin, D. Ivanov, R. Kazakov. "Raspredelenie temperatury v sterzhne s dvustoronnim podvodom tepla". Izvestija tomskogo tehnicheskogo universiteta, vol. 321, no. 4, pp. 5-6, 2012.

[12] V. Tonkonogij. "Avtomatizacija tehnologicheskogo processa nanesenija ionno-plazmennyh iznosostojkih pokrytij na rezhushij instrument.” D.Science. thesis, ONPU, Odessa, 2004.

[13] A. Stopakevych, Systemnyi analyz y teoryia slozhnykh system upravlenyia, Odessa: Astroprynt, 2013.

[14] V. Lunev, V. Samoylov. "Balans jenergij i teplovye jeffekty pri metallizacii sverhtverdyh materialov kondensaciej plazmy". Sverhtverdye materialy, no. 2, pp. 7-12, 1980.

[15] B. Levytan, I. Sargsyan. Operatory Shturma — Liuvillja i Diraka. Moscow: Nauka, 1988.

УДК 681.586.5

\title{
ПРИМЕНЕНИЕ ВОЛОКОННО-ОПТИЧЕСКИХ ДАТЧИКОВ В СИСТЕМАХ АВТОМАТИЧЕСКОГО ДИАГНОСТИРОВАНИЯ СУДОВЫХ ГАЗОТУРБИННЫХ УСТАНОВОК
}

\author{
Сандлер А.К. ${ }^{1}$, Карпилов А.Ю. ${ }^{2}$ \\ ${ }^{1,2}$ Национальный университет "Одесская морская академия", г. Одесса, Украина \\ E-mail: ${ }^{1}$ albertsand4@gmail.com, ${ }^{2}$ kau.onma@gmail.com
}

Copyright (C) 2018 by author and the journal "Automation technologies and business - processes. This work is licensed under the Creative Commons Attribution International License (CC BY). http://creativecommons.org/licanses/by/4.0

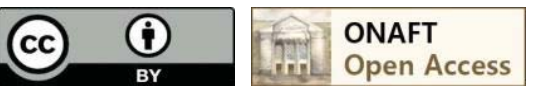

DOI:

Аннотация: Нарушение режимов смазки неизбежно инициирует зарождение прогрессирующих с высокой скоростью дефектов в элементах подшипниковых узлов. Несвоевременное обнаружение деструктивных проиессов в подиипниках роторов газотурбинных установок приводит к полному выходу установки из строя и может иметь катастрофические последствия, как для судна, так и группы судов в сложных навигационных условиях. $K$ сожалению, существующие типь акселерометров в условиях кониентрированного воздействия мощньх электромагнитных, термических и вибрационных полей, компактно расположенного судового оборудования, не обеспечивают эффективный контроль вибрации подиипников в наиболее информативном высокочастотном 
диапазоне. В сложившейся ситуации наиболее целесообразным может оказаться использование волоконнооптических измерительных преобразователей на основе квариевого стекла, как материала наиболее стойкого к больиинству эксплуатационных дестабилизирующих факторов. Для детектирования и измерения уровня вибрации подшипников газотурбинных установок могут использоваться волоконно-оптические акселерометры, построеннье по схемам поляризационной, фазовой и амплитудной модуляции. В тоже время анализ показал, что возможности существующих волоконно-оптических устройств недостаточны для эффективного контроля вибрации в специальных условиях. В создавщейся ситуации представляется рациональной рекомендация акцентировать внимание на компенсации деградации свойств чувствительного элемента акселерометра и прилегающих линий связи. Рекомендуемое схемотехническое решение представляет собой волоконный акселерометр амплитудной модуляиии, состоящчй из герметичного корпуса, опорного световода, содержащзего блок C/G- линз, измерительного и компенсирующего световодов. Световоды имеют отражающие слои из сапфирового стекла на ториах и эксиентриковую массу из квариевого стекла на измерительном световоде. Главное отличие рекомендованного акселерометра от ранее известных конструкций заключается в том, что световоды имеют депрессированную сердиевину, оптические фильтры включены в состав измерительного и компенсирующего световодов, разъединение/объединение излучения осуществляется в блоке $C / G$-линз, а для компенсации температурного влияния используется жестко соединенная с опорным световодом биметаллическая пластина.

Abstract: The violation of lubrication regimes inevitably initiates the emergence of defects that are progressive at a high speed in the elements of bearing units. Failure to detect destructive processes in the bearings of the rotors of gas turbine installations leads to complete installation failure and can have disastrous consequences for both the vessel and the group of vessels in difficult navigation conditions. Unfortunately, the existing types of accelerometers under conditions of concentrated exposure to powerful electromagnetic, thermal and vibration fields, compactly located shipboard equipment, do not effectively control the vibration of bearings in the most informative high-frequency range. In this situation, the most appropriate may be the use of fiber optic transducers based on silica glass, as the material most resistant to most operational destabilizing factors. For the detection and measurement of the vibration level of bearings in gas turbine installations, fiber optic accelerometers can be used, constructed according to polarization, phase and amplitude modulation schemes. At the same time, the analysis showed that the capabilities of existing fiber-optic devices are insufficient to effectively control the vibration in special conditions. In this situation, it seems a rational recommendation to focus attention on compensating for the degradation of the properties of the accelerometer's sensitive element and adjoining communication lines. The recommended circuit solution is a fiber accelerometer of amplitude modulation, consisting of a sealed housing, a reference fiber containing a block of $C$ / $G$ lenses, measuring and compensating fibers. The fibers have reflective sapphire crystal layers on the ends and an eccentric mass of quartz glass on the measuring light guide. The main difference between the recommended accelerometer and previously known designs is that the optical fibers have a depressed core, optical filters are included in the measuring and compensating optical fibers, separation / integration of radiation is carried out in the C / G-lens unit, and to compensate for temperature effects bimetallic plate connected to the reference light guide.

Ключевые слова: вибрация, волоконно-оптический, акселерометр, термостабильность.

Keywords: vibration, fiber optic, accelerometer, thermal stability

\section{Введение}

Постоянно изменяющиеся в течение длительных периодов времени гидродинамические и климатические условия эксплуатации пропульсивного комплекса судна, в сочетании с влиянием агрессивной морской среды, вызывают резкое снижение ресурса смазочных материалов, использующихся в газотурбинных установках (ГТУ). Нарушение режимов смазки неизбежно инициирует зарождение прогрессирующих с высокой скоростью дефектов в элементах подшипниковых узлов. Несвоевременное обнаружение деструктивных процессов в подшипниках роторов ГТУ приводит к полному выходу установки из строя и может иметь катастрофические последствия, как для судна, так и группы судов в сложных навигационных условиях.

К сожалению, существующие типы акселерометров в условиях концентрированного воздействия мощных электромагнитных, термических и вибрационных полей, компактно расположенного судового оборудования, не обеспечивают эффективный контроль вибрации подшипников в наиболее информативном высокочастотном диапазоне.

В сложившейся ситуации наиболее целесообразным может оказаться использование волоконно-оптических измерительных преобразователей на основе кварцевого стекла, как материала наиболее стойкого к большинству эксплуатационных дестабилизирующих факторов (ДФ).

В то же время в научно-технической литературе недостаточно отражены особенности создания волоконнооптические акселерометры (BОА) для систем технического диагностирования, эксплуатируемых в условиях концентрированного воздействия ДФ, соответственно не рассматриваются конструктивно-технологические особенности, и не дается оценка их метрологических и эксплуатационных характеристик в данных условиях.

Анализ литературных источников и постановка задачи

Рост температуры подшипников ГТУ обусловлен "внутренним" нагревом подшипника и внешним подводом тепла. 
"Внутренний" нагрев подшипников качения вызывается упругой деформацией тел качения и беговых дорожек колец при приложении к ним нагрузок. При этом происходит деформация нагружаемых участков и последующее возвращение их в исходное состояние. Внутреннее трение между частицами металла в таком процессе приводит к выделению тепла. Кроме того, подшипники, расположенные в "горячих зонах" двигателя (в районе камеры сгорания и турбины), испытывают значительный дополнительный подогрев от горячих деталей, что приводит к необходимости организации повышенного теплосъема с помощью прокачиваемого масла. В этих условиях температура для подшипников качения ГТУ достигает границ в $225 \ldots 250{ }^{\circ} \mathrm{C}[1]$.

Для детектирования и измерения уровня вибрации подшипников ГТУ могут использоваться ВОА, построенные по схемам поляризационной, фазовой и амплитудной модуляции. Во всех типах волоконных акселерометров температурные воздействия на оптический волновод (OB) такого уровня, приводя к изменению его параметров, могут влиять одновременно на одну или сразу несколько характеристик оптической волны.

Поляризационные датчики отличаются от амплитудных и интерферометрических наличием поляризаторов и анализаторов. Эти элементы, как правило, должны устанавливаться в месте получения первичной информации, что связано с возможной деполяризацией излучения или дополнительным двулучепреломлением, которое могут внести подводящие и отводящие волоконные световоды. Такое расположение поляризаторов, изготавливаемых обычно на основе органических пленок, существенно ограничивает область применения поляризационных датчиков, снижает верхний предел рабочих температур до $50 \ldots 60^{\circ}$ С. Ограничение по верхнему пределу рабочих температур делает нецелесообразным применение поляризационных ВОА для задач диагностирования ГТУ.

ВОА фазовой модуляции строятся по наиболее распространенным схемам на основе гомодинного и гетеродинного интерферометра Маха - Цендера.

Акселерометры этого типа обычно реализуются по дифференциальной схеме: лазерное излучение разделяется полупрозрачным зеркалом на два потока, один из которых направляется в ОВ, локализованное в зоне измерения. Другой поток излучения пропускается через опорное ОВ, вне зоны воздействия измеряемой величины. В измерительном преобразователе реализован принцип полной "асимметрии" измерительного и опорного канала относительно не только информативной измеряемой величины, но и относительно ДФ и нелинейных составляющих функции преобразования.

Информационный сигнал в каждом из плечей интерферометра зависит от изменений постоянной распространения волны $\beta$ и длины OB $L$, находящегося под действием контролируемой величины. Изменения $\Delta \beta$ зависит от разности показателей преломления сердцевины и оболочки OB $\Delta n$, а также от текущего радиуса сердцевины $\rho$.

Генерируемый температурным воздействием фазовый сдвиг на единицу длины ОВ в гомодинных интерферометрах определяется как [2]

$$
\frac{d \varphi}{L d T}=k_{0}\left(\frac{1}{k_{0}} \frac{\partial\left(k_{0} n\right)}{\partial \rho} \frac{\partial \rho}{\partial T}+\frac{\partial n}{\partial T}+\frac{n}{L} \frac{\partial L}{\partial T}\right),
$$

где $\kappa_{0}-$ волновое число, связанное с постоянной распространения соотношением $\beta=\kappa_{0} n$.

Оценку температурного влияния на работу гомодинных ВОА можно выполнить на основе общепринятых данных $\partial a / \partial T$ и $\partial n / \partial T: \partial a / \partial T=3 \cdot 10^{-5} \mathrm{~K}^{-1} ; \partial n / \partial T=-6,1 \cdot 10^{-5} /{ }^{0} \mathrm{C}$. Основные величины температурных изменений материала ОВ представлены в таблице $1[3]$.

Таблица 1 - Температурные изменения в материалах $\mathrm{AgCl} / \mathrm{AgBr}$

\begin{tabular}{|c|c|c|c|c|c|c|}
\hline$T,{ }^{0} \mathrm{C}$ & 0 & 50 & 100 & 150 & 200 & 250 \\
\hline$\Delta n(T)$ & 0 & $-3,05 \cdot 10^{-3}$ & $-6,1 \cdot 10^{-3}$ & $-9,15 \cdot 10^{-3}$ & $-0,0122$ & $-0,0153$ \\
\hline$n_{1}$ & 2,1106 & 2,1076 & 2,1045 & 2,10145 & 2,0984 & 2,09535 \\
\hline$\Delta a(T)$ & 0 & $1,5 \cdot 10^{-3}$ & $3 \cdot 10^{-3}$ & $4,5 \cdot 10^{-3}$ & $6,0 \cdot 10^{-3}$ & $7,5 \cdot 10^{-3}$ \\
\hline$a(T)$, мкм & 30,00 & 30,045 & 30,09 & 30,135 & 30,180 & 30,225 \\
\hline
\end{tabular}

Из последней строки таблицы 1 видно, что увеличение диаметра при температурах вплоть до $T=200^{0} \mathrm{C}$ составляют малую величину $(0,7 \%)$, так что для одномодового режима распространения такое увеличение радиуса сердцевины не вносит заметных изменений.

Напротив, расчеты показали, что изменения $\Delta h(T)$ оказывают ощутимое влияние на характеристики моды. Результаты расчетов постоянной распространения $\beta$ при нагревании для волокна $\mathrm{AgClBr}$ приводятся на рис. 1, 2 .

Эффект изменений является, в основном, следствием значительного уменьшения показателя преломления $n_{1}=$ $2,1106 \ldots 2,09535$ при возрастании температуры в диапазоне $T=0 \ldots 250^{\circ} \mathrm{C}$. На рис. 1 показано уменьшение значений постоянных распространения фундаментальной моды одномодового волокна для трех значений температуры. На рис. 2 представлен сдвиг величин постоянной распространения с температурой фундаментальной моды $\Delta \beta_{10}=0,00904$ мкм $^{-1}$, что меньше, чем те же изменения для высших мод, например $\Delta \beta_{11}=0,00924$ мкм $^{-1}$ при температурах $0 \ldots 250^{0} \mathrm{C}$ [3]. 
Тогда для ОВ с внутренним напряжением и $\Delta n=2 \cdot 10^{-4}$ получаем $d\left(\Delta \beta_{\mathrm{s}}\right) / L \Delta T=2,5$ рад/м·К, а для световода с анизотропией, созданной эллиптичностью жилы с $\Delta n=8,5 \cdot 10^{-4}, d(\Delta \beta \ni) / L \Delta T=3,5 \cdot 10^{-3}$ рад/м·К [4].

В отличие от гомодинного, оптическое гетеродинное детектирование фазовой модуляции использует периодическое изменение интенсивности излучения, падающего на фотодетектор с частотой, равной разности распространяющихся по плечам интерферометра частот излучения. Разностный сигнал на выходе фотодетектора несет ту же информацию о фазовой модуляции, что и излучение в измерительном ОВ.

ВОА этого типа рассматриваются как системы, в которых осуществлен принцип "симметрии" измерительных каналов относительно влияющих факторов и нелинейных составляющих функций преобразования и "асимметрии" относительно информативных измеряемых величин. Соответственно в структуре преобразователей организуется два измерительных канала, "симметричных" относительно воздействия влияющих факторов и "асимметричных" относительно информативной измеряемой величины. Реализуются инвариантные параметрические преобразователи данного типа на основе интерферометра Маха-Цендера с ОВ в одном плече и ячейкой Брэгга в другом.

Фазовая чувствительность к воздействию температуры на единицу длины ОВ определяется чувствительностью к изменению длины $L$ и показателя преломления $n[2]$

$$
\frac{1}{L} \frac{d(\Delta \varphi)}{d T}=\frac{k_{0}}{L}\left[L \frac{\Delta n}{n} \frac{d n}{d T}+\Delta n \frac{d L}{d T}+L \frac{\Delta n T_{c}}{T_{c}-T_{\mathrm{II}}}\right] .
$$

где $T_{c}$ - температура окружающей среды;

$T_{\text {пл }}$ - температура плавления материала оболочки.

Использование данных [3] при расчетах показывает, что в гетеродинных измерительных преобразователях может быть достигнута термочувствительность $d(\Delta \beta) / L \Delta T=0,85 \ldots 0,95$ рад/м·К.

Для наиболее распространенных ВОА амплитудного типа термостабильность зависит от изменения геометрии оптического канала между передающим и принимающим OB, а также от качества открытых торцовых поверхностей световодных элементов измерительного преобразователя.

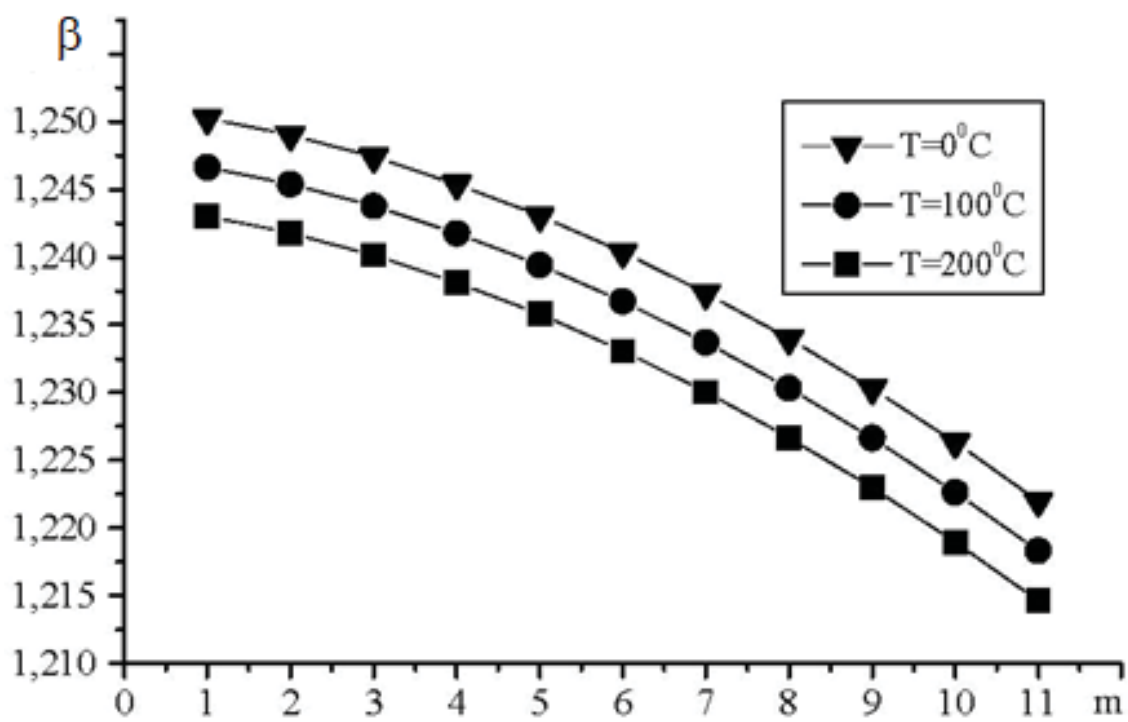

Рис. 1 - Постоянные распространения $\beta$ мод ОВ $\mathrm{AgClBr}$ с параметрами: $2 a=100$ мкм, $n_{1}-n_{2}=0,05$; для холодного состояния волокна $(T=0)$, и нагретого на 100 и $200^{\circ} \mathrm{C}$.

В амплитудных ВОА изменение геометрии оптического канала между световодными элементами достигается за счет:

рассогласования диаметров и апертур;

поперечного смещения;

продольного смещение;

углового смещение.

В первом случае, когда два волокна различного диаметра/апертур объединены и волокна аксиально идентичны. Коэффициент передачи устанавливается из соотношений

$$
a_{d}=-10 \log \left(\frac{d}{D}\right)^{2} a_{N A}=-10 \log \left(\frac{N A_{R}}{N A_{T}}\right)^{2},
$$

где $d, D$ - диаметры сопрягаемых ОВ;

$N A_{R}, N A_{T}$ - апертуры сопрягаемых OB. 


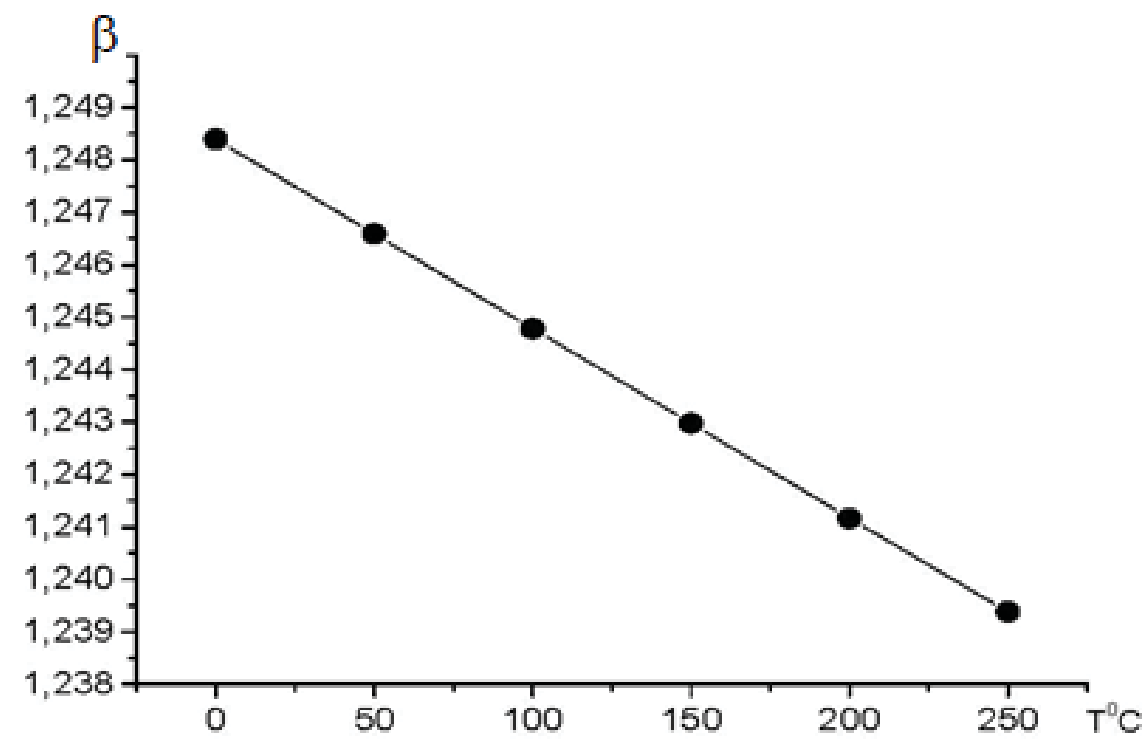

Рис. 2 - Температурный сдвиг постоянной распространения $\beta_{01}(T)$, мкм ${ }^{-1}$, для одномодового $\mathrm{AgClBr}$ волокна

Для случая поперечного смещения для ОВ со ступенчатым профилем показателя преломления коэффициент передачи может быть определен как

$$
a_{\text {os }}=-10 \log \left\{\frac{2}{\pi} \operatorname{arcos}\left(\frac{e}{2 \rho}\right)-\frac{e}{\pi \rho}\left[1-\left(\frac{e}{2 \rho}\right)^{2}\right]^{0.5}\right\}
$$

где $\rho$ - радиусы сопрягаемых ОВ;

$e$ - величина смещения осей ОВ.

Коэффициент передачи при угловом смещении осей волокон может быть выражен для ОВ со ступенчатым профилем показателя преломления как

$$
a_{U S}=-10 \log \left\{\frac{2}{\pi}\left[\operatorname{arctg}\left(\frac{\sin ^{2} \theta_{c}}{\sin ^{2} \frac{\alpha}{2}}-1\right)^{0.5}-\frac{\sin ^{2} \frac{\alpha}{2}}{\sin ^{2} \theta_{c}}\left(\frac{\sin ^{2} \theta_{c}}{\sin ^{2} \frac{\alpha}{2}}-1\right)^{0.5}\right]\right\} \text {, }
$$

где $\Theta_{\mathrm{c}}-$ апертурный угол;

$\alpha-$ угол между осями ОВ.

Коэффициент передачи при продольном смещении осей волокон может быть выражен для ОВ со ступенчатым профилем показателя преломления как

где $l$ - расстояние между торцами ОВ [5].

$$
a_{P S}=-10 \log \left(1-\frac{4 l}{3 \pi \rho} \operatorname{tg} \theta_{c}\right)
$$

Расчет приращений коэффициентов передачи для всех случаев изменения геометрии оптического канала, с учетом данных [3], показывает, что в интервале температур $100 \ldots 200^{\circ} \mathrm{C}$, может быть достигнута термочувствительность на порядок выше, чем у фазовых измерительных преобразователей. Однако, на практике эта величина на порядок больше.

Это обусловлено воздействием на кварцевое стекло элементов акселерометра гидролитической, кислотной и щелочной коррозии, интенсивность которых экспоненциально увеличивается с температурой. В результате термофлуктуационного и гидролитического воздействия ДФ происходит:

разрушения силоксановых мостиковых связей в поверхностном слое стекла толщиной 30 ... 120 мкм;

нарушение геометрии расположения волноводных элементов датчика;

образование "паразитного" слоя на элементах датчика.

Кроме того, подтверждена связь размеров волоконных элементов и вероятности того, что на их поверхности или в объеме могут зарождаться опасные дефекты, резко снижающие оптические и механические свойства кварцевого стекла [6].

Таким образом, в ВОА с открытым оптическим каналом, при рассеянии света на входном торце ОВ возбуждение волноводных мод происходит в более широком интервале углов падения плоской электромагнитной волны, что 
эквивалентно увеличению апертурного угла ввода.

Это явление имеет простую физическую интерпретацию: при диффузном рассеянии на входном торце ОВ в угловую апертуру попадает рассеянное излучение при углах ввода, превышающих апертурный угол. Угловые характеристики ввода ОВ с торцевым рассеивателем аналогичны характеристикам ввода ОВ с нерассеивающим торцом, но имеющим больший апертурный угол и меньшую эффективность ввода [7].

Как итог, термочувствительность ВОА оказывается сравнима с фазовыми, нивелируя все преимущества амплитудных измерительных преобразователей.

Цель и задачи исследования

В создавшейся ситуации представляется рациональной рекомендация акцентировать внимание на компенсации деградации свойств чувствительного элемента акселерометра и прилегающих линий связи.

Рекомендуемое схемотехническое решение представляет собой волоконный акселерометр амплитудной модуляции, состоящий из герметичного корпуса, опорного световода, содержащего блок $C / G$ - линз, измерительного и компенсирующего световодов (рис. 3). Световоды имеют отражающие слои из сапфирового стекла на торцах и эксцентриковую массу из кварцевого стекла на измерительном световоде. Главное отличие рекомендованного акселерометра от ранее известных конструкций заключается в том, что световоды имеют депрессированную сердцевину, оптические фильтры включены в состав измерительного и компенсирующего световодов, разъединение/объединение излучения осуществляется в блоке $C / G$ - линз, а для компенсации температурного влияния используется жестко соединенная с опорным световодом биметаллическая пластина.

\section{Результаты исследований}

В статическом режиме (механические колебания отсутствуют), в элементах акселерометра происходит уменьшение интенсивности оптического излучения, которое обусловлено только влиянием дестабилизирующих неконтролируемых факторов. В этом режиме, под влиянием температуры, меняется геометрия биметаллической пластины, зафиксированной на участке опорного световода. Увеличение радиуса изгиба опорного световода вызывает определенное уменьшение потерь мощности оптического излучения пропорционально температуре внешней среды. Таким образом, в пределах режима происходит термокомпенсация повышенной температуры.

В динамическом режиме (контроль механических колебаний) под влиянием вибрации происходит движение эксцентриковой массы, который превращается в деформацию сдвига в измерительном световоде. При таких условиях меняется соотношение показателей преломления сердцевины и оболочки измерительного световода, что вызывает адекватное нарушение в нем условия полного внутреннего отражения света. То есть, часть излучения, введенное в измерительный световод, будет выводиться за его границы. Оставшаяся часть света, будет отражаться от отражающего слоя, и возвращаться по опорному световоду к фотоприемнику.
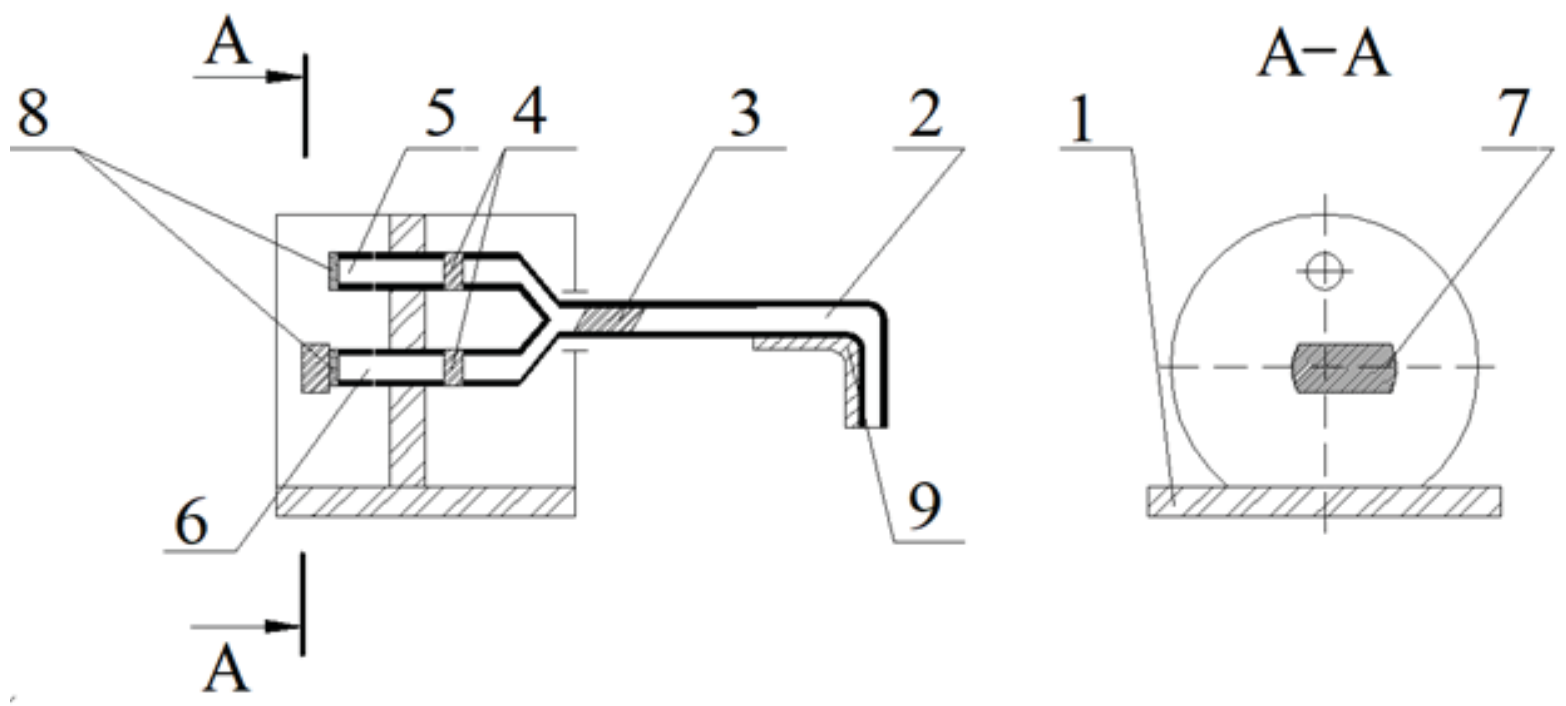

Рис. 3. - Волоконно-оптический акселерометр: 1 - корпус; 2 - опорный световод; 3 - $C / G$ - линзы; 4 оптический фильтр; 5 - измерительный световод; 6 - компенсирующий световод; 7 - эксцентриковая масса; 8 - отражающий слой; 9 - биметаллическая пластина

Излучение, которые поступает от измерительного и компенсирующего световодов, благодаря комбинации $C / G$ линз и оптических фильтров имеет разные длины волн. В первом динамическом субрежиме фотоприемником регистрируется излучения с длиной волны, которая поступает от компенсирующего световода и учитывается в 
дальнейшем как поправка. Во втором динамическом субрежиме фотоприемником регистрируется излучение с длиной волны, которая поступает от измерительного световода.

Дальнейшая обработка величины интенсивности излучения, которое регистрируется поочередно в двух субрежимах, позволит получить электрический сигнал, пропорциональный величине измеренной вибрации и в котором будет компенсирована составляющая от влияния дестабилизирующих факторов.

\section{Выводы}

Рекомендованное схемотехническое решение волоконно-оптического акселерометра, благодаря комбинации оптических элементов обеспечит:

- $\quad$ более адекватного преобразования параметров вибрации в изменения информационного сигнала;

- компенсации влияния дестабилизирующих факторов на измерительный канал акселерометру;

- приближение акселерометру, благодаря компенсации дестабилизирующих факторов, к источнику возникновения вибрации в контролируемых узлах;

- повышение качества функционирования за счет избрания рациональной схемы модуляции излучения;

- упрощение конструктивного исполнения.

В целом, использование предлагаемого устройства позволит адекватно и достоверно оценивать показатели вибрации подшипниковых узлов судовых газотурбинных установок.

\section{Список использованных источников}

[1] Иноземцев, А.А., Нихамкин, М.А., Сандрацкий, В.Л. Основы конструирования авиационных двигателей и энергетических установок. - М.: Машиностроение, 2007. - 208 с

[2] Бусурин, В.И., Носов, Ю.Р. Волоконно-оптические датчики. Физические основы, вопросы расчета и применения. - М.: Энергоатомиздат, 1990. - 256 с.

[3] Tulaikova, T. Fiber-optical sensors for ecology usage. - LAP-LAMBERRT Academic Publishing, 2011. - 205 p.

[4] Гуляев, Ю.В., Меш, М.Я., Проклов, В.В. Модуляционные эффекты в волоконных световодах и их применение. М.: Радио и связь, 1991, 152 с.

[5] Влияние параметров волокна на качество сращивания. [Электронный ресурс]. Режим доступа optokon.ua/arts/liyanie-parametrov-volokna-na-kachestvo-srashchivaniya.

[6] Сандлер, А.К., Логишев, И.В., Сандлер, А.А. Структурная модель инвариантного волоконного акселерометра. // Судовые энергетические установки. - Одесса: ОНМА. - 2011. - Вып. 25. - С. 87 - 93.

[7] Кизеветтер, Д.В., Малюгин, В.И. Влияние дефектов торцевой поверхности световода на эффективность ввода излучения // Журнал технической физики. - 2002. - Т. 72. - №. 9. - С. 80 - 86.

\section{References}

[1] Inozemtsev, A.A., Nikhamkin, M.A., Sandratskiy, V.L.(2007). Osnovy konstruirovaniya aviatsionnykh dvigateley i energeticheskikh ustanovok [Basics of designing aircraft engines and power plants]. Moscow: Mashinostroyeniye [in Russian].

[2] Busurin, V.I., Nosov, YU.R.(1990). Volokonno-opticheskiye datchiki. Fizicheskiye osnovy, voprosy rascheta i primeneniya [Fiber optic sensors. Physical basis, questions of calculation and application]. Moscow: Energoatomizdat [in Russian].

[3] Tulaikova, T. Fiber-optical sensors for ecology usage. - LAP-LAMBERRT Academic Publishing, 2011. - 205 p.

[4] Gulyayev, YU.V., Mesh, M.YA., Proklov, V.V.(1991). Modulyatsionnyye effekty v volokonnykh svetovodakh i ikh primeneniye. [Modulation effects in optical fibers and their application] Moscow: Radio i svyaz' [in Russian].

[5] Sait kompaniya "OPTOKON a.s." [Site company "OPTOKON a.s."] http:www.optokon.ua. Retrieved from optokon.ua/arts/liyanie-parametrov-volokna-na-kachestvo-srashchivaniya.

[6] Sandler, A.K., Logishev, I.V., Sandler, A.A. (2011). Strukturnaya model' invariantnogo volokonnogo aksele-rometra [Structural model of an invariant fiber accelerometer]. Sudovyye energeticheskiye ustanovki - Ship power plants. 25, 87 - 93 [in Ukraine].

[7] Kizevetter, D.V., Malyugin, V.I. (2002). Vliyaniye defektov tortsevoy poverkhnosti svetovoda na effektivnost' vvoda Izlucheniya [Effect of defects on the end surface of the fiber on the efficiency of radiation input]. Zhurnal tekhnicheskoy fiziki - Technical Physics Journal, T. 72, 9, 80 - 86 [in Russian]. 